\title{
New LMI-based Criteria for Lagrange Stability of Cohen-Grossberg Neural Networks with General Activation Functions and Mixed Delays
}

\author{
Xiaohong Wang ${ }^{1}$, Huan $\mathbf{Q i}^{2}$ \\ ${ }^{1}$ Department of Control Science and Engineering, Huazhong University of Science and Technology, \\ 1037 Luоyи Road, \\ Wuhan, 430074, China \\ E-mail: wxhong2006@163.com \\ 2 Department of Control Science and Engineering, Huazhong University of Science and Technology, \\ 1037 Luоуи Road, \\ Wuhan, 430074, China \\ E-mail: qihuanster@gmail.com \\ Received 10 April 2012 \\ Accepted 19 February 2013
}

\begin{abstract}
In this paper, the problem on Lagrange stability of Cohen-Grossberg neural networks (CGNNs) with both mixed delays and general activation functions is considered. By virtue of Lyapunov functional and Halanay delay differential inequality, several new criteria in linear matrix inequalities (LMIs) form for the global exponential stability in Lagrange sense of CGNNs are obtained. Meanwhile, the limitation on the activation functions being bounded, monotonous and differentiable is released, which generalizes and improves those existent results. Moreover, detailed estimations of the globally exponentially attractive sets are given out. It is also verified that outside the globally exponentially attractive set, there is no equilibrium state, periodic state, almost periodic state, and chaos attractor of the CGNNs. Finally, two numerical examples are given to demonstrate the theoretical results.
\end{abstract}

Keywords: Cohen-Grossberg neural networks; Lagrange stability; Globally exponentially attractive set; Linear matrix inequality(LMI); Time-varying delays and finite distributed delays

\section{Introduction}

Since the discovery of the Cohen-Grossberg neural networks (CGNNs) [1] in 1983, a lot of applications have been appeared in many fields to solve control, signal processing, associative memory, parallel computation and nonlinear optimization problems. In employing CGNNs to solve these problems, one of the most desirable properties of CGNNs is the Lyapunov stability. From a dynamical system point of view, globally stable networks in Lyapunov sense are monostable systems, which have a unique equilibrium attracting all trajectories asymptotically. A large body of research now exists on the study of globally stable in Lyapunov sense for CGNNs, more specific results are referred to [2-11]. In many other applications, however, monostable neural networks have been found computationally restrictive and multistable dynamics are essential to deal with important neural computations desired. In these cir-

\footnotetext{
*Corresponding author. Xiaohong Wang, E-mail: wxhong2006@163.com.
} 
cumstances, neural networks are no longer globally stable and more appropriate notions of stability are need to deal with multistable systems. In this context, many researchers focus on the Lagrange stability.

It is worth to mention that unlike Lyapunov stability, Lagrange stability refers to the stability of the total system, rather than the stability of the equilibriums, because the Lagrange stability is considered on the basis of the boundedness of solutions, which depend on the existence of global attractive sets (see $[12,18,19,21-25])$. We also note that Lagrange stability has attracted phenomenal worldwide attention. In [13], Rekasius considered asymptotic stability in Lagrange sense for nonlinear feedback control systems. In [14], Lagrange stability was discussed by Thornton and Mulholland as a useful concept for determining the stability of ecological systems. Soon, Passino and Burgess [15] adapt the concept of Lagrange stability to investigate discrete event systems. See also $[16,17]$ for recent results on Lagrange stability for pendulum-like systems.

In recent years, Lagrange stability has been further researched into various kinds of neural networks. However, it should be noted that, in hardware implementation, time delays occur due to finite switching speed of the amplifiers and communication time. The time delays may lead to oscillation, divergence, or instability, which may be harmful to a system. On the other hand, it has also been shown that the process of moving images requires the introduction of delay in the signal transmitted through the networks. Therefore, the study of Lagrange stability of neural networks with delays is practically required, and it has been extensively studied. For example, in [18, 19], Liao et al. apply Lyapunov functions to study Lagrange stability for recurrent neural networks with constant time delays and time-varying delays. In [20], Yang and Cao consider stability in Lagrange sense of a class of feedback neural networks for optimization problems. Then [21] and [22] continued to probe further into the Lagrange stability for neutral type and periodic recurrent neural networks along the methods in $[18,19]$, respectively. In [24, 25], Lagrange stability is discussed for Cohen-Grossberg neural net- works (CGNNs) with time-varying delays and finite distributed delays. To our best knowledge, these results of Lagrange stability analysis for neural networks depend mainly on Lyapunov-like functions, and there are few results made on it by LMIs [32].

Moreover, in conducting stability analysis of a neural network, the conditions to be imposed on the neural network are determined by the characteristics of activation function as well as network parameters. As we know, when neural networks are designed for problem solving, it is desirable for their activation functions to be general. To facilitate the design of neural networks, it is important that the neural networks with general activation functions are studied. The generalization of activation functions will provide a wider scope for neural network designs and applications. So, in [23], Tu et al. study the Lagrange stability for recurrent neural networks with general activation functions and timevarying delays. At present, although a series of results for Cohen-Grosseberg neural networks are obtained (see [2-12, 24, 25]), However, most of the existing results about CGNNs focus on the Lyapunov stability analysis. Up to now, there is no reported result, to our best knowledge, dealing with the global Lagrange exponential stability for CGNNs with general activation functions and finite distributed delays and time-varying delays. Especially, the criteria of Lagrange stability for CGNNs by means of linear matrix inequalities (LMIs) also do not appear at all. Hence this field remains important and challenging.

Motivated by the above discussion, the objective of this paper is to study the global exponential stability in Lagrange sense and estimate the size of globally exponentially attractive sets for the addressed CGNNs with general activation functions and mixed time delays. Different from most of the previous conclusions, the results are performed in LMIs, which will be efficiently solved by the Matlab LMI Toolbox [26]. The main innovative points are as follows:

(I) The methodology developed in [27] will be extended to study the Lagrange stability for general Cohen-Grossberg neural networks with timevarying and finite distributed delays (1), which will be introduced in the next section. Meanwhile, the 
globally exponentially attractive sets are also obtained. The conclusion contains some existing results as its special cases.

(II) When studying the properties of Lagrange stability for neural network systems, people usually assume that their activation functions are monotonic, or differentiable, even or bounded. However, the activation function discussed in this paper is neither bounded, nor monotonous nondecreasing. What is more, with regard to time delay, it is only need to bounded, not considering its differential form.

(III) In addition, as a direct application of the results, it will be obtained later by a further theorem.

In the next section, some preliminaries, including some necessary notations, definitions, assumptions and lemmas are described. The main results and their corresponding proofs for CGNNs to have globally exponentially attractive sets and positive sets are given in Section 3. What's more, an application is present in Section 4 and finally a summery is demonstrated give in Section 5.

Notations: Throughout this paper, the symbols $R^{n}$ and $R^{n \times m}$ stand, respectively, for the $n$ dimensional Euclidean space and the set of all $n \times m$ real matrices. $A^{T}$ and $A^{-1}$ denote the matrix transpose and matrix inverse. $A>0$ or $A<0$ denotes that the matrix $A$ is a symmetric and positive definite or negative definite matrix. Meanwhile, $A<B$ indicates $A-B<0$ and $\|*\|$ is the Euclidean vector norm. Moreover, in symmetric block matrices, we use "*" as an ellipsis for the terms that are introduced by symmetry.

\section{Model Description and Preliminaries}

Considering the following CGNNs model with timevarying delays and finite distributed delays:

$$
\begin{array}{r}
\dot{x}(t)=\alpha(x(t))[-D x(t)+A g(x(t))+B g(x(t-\tau(t))) \\
\left.+C \int_{t-\sigma(t)}^{t} g(x(s)) d s+U\right],
\end{array}
$$

where $x(t)=\left(x_{1}(t), \ldots, x_{n}(t)\right)^{T}$ and $x_{i}(t)$ is the state variable associated with the neuron. $\alpha(x(t))=\operatorname{diag}\left\{\alpha_{1}\left(x_{1}(t)\right), \ldots, \alpha_{n}\left(x_{n}(t)\right)\right\}$ and $\alpha_{i}$ is an appropriately amplification function. $D=$ $\operatorname{diag}\left\{d_{1}, \ldots, d_{n}\right\}$ and $d_{i}$ denotes the behaved function. $g(x(t))=\left(g_{1}\left(x_{1}(t)\right), \ldots, g_{n}\left(x_{n}(t)\right)\right)^{T}, g(x(t-$ $\tau(t)))=\left(g_{1}\left(x_{1}\left(t-\tau_{1}(t)\right)\right), \ldots, g_{n}\left(x_{n}\left(t-\tau_{n}(t)\right)\right)\right)^{T}$. The activation function $g_{j}$ shows how the neurons respond to each other. $\int_{t-\sigma(t)}^{t} g(x(s)) d s=$ $\left(\int_{t-\sigma_{1}(t)}^{t} g_{1}\left(x_{1}(s)\right) d s, \ldots, \int_{t-\sigma_{n}(t)}^{t} g_{n}\left(x_{n}(s)\right) d s\right)^{T}$. The time-varying delay $\tau(t)=\left(\tau_{1}(t), \ldots, \tau_{n}(t)\right)^{T}$ satisfies $0 \leqslant \tau_{i}(t) \leqslant \tau_{i}$, and the finite distributed delay $\sigma(t)=\left(\sigma_{1}(t), \ldots, \sigma_{n}(t)\right)^{T}$ satisfies $0 \leqslant \sigma_{i}(t) \leqslant \sigma_{i}$, here $\tau_{i}$ and $\sigma_{i}$ are constants. $A=\left(a_{i j}\right), B=\left(b_{i j}\right), C=$ $\left(c_{i j}\right) \in R^{n \times n}$ tell us how the neurons are connected in the network. $U=\left(U_{1}, \ldots, U_{n}\right)^{T}$ and $U_{i}$ is the input. Function $\alpha_{i}$ is continuous and satisfies $0<\alpha_{i}^{-} \leqslant \alpha_{i}(\cdot) \leqslant \alpha_{i}^{+}$.

Here, let $\tau=\max _{1 \leqslant i \leqslant n} \tau_{i}$, and $\sigma=\max _{1 \leqslant i \leqslant n} \sigma_{i}$. $C[X, Y]$ is a class of continuous mapping set from the topological space $X$ to the topological space $Y$. Especially, $C=\left[[-h, 0], R^{n}\right]$, where $h=\max \{\tau, \sigma\}$. For any initial function $\varphi(s) \in C, s \in\left[t_{0}-h, t_{0}\right]$, the solution of (1) that starts from the initial condition $\varphi$ will be denoted by $x\left(t, t_{0}, \varphi\right)$ or simply $x(t)$ if no confusion should occur.

Throughout this paper, we make the following assumption:

(A) There exist two diagonal matrices $L=$ $\operatorname{diag}\left\{\mathrm{L}_{1}, \cdots, \mathrm{L}_{\mathrm{n}}\right\}$ and $F=\operatorname{diag}\left\{\mathrm{F}_{1}, \cdots, \mathrm{F}_{\mathrm{n}}\right\}$ such that the following inequalities hold:

$$
L_{i} \leqslant \frac{g_{i}(x)-g_{i}(y)}{x-y} \leqslant F_{i}
$$

for all $x, y \in R, x \neq y, i=1,2, \cdots, n$.

Remark 1. In the literature $[24,25]$, the results were obtained under the condition that the timevarying delays are continuously differentiable, of which the derivative was bounded and smaller than one, and the activation functions were limited on bounded and monotonically non-decreasing. It is needed to point out that, in this paper, the presented results do not need the conditions mentioned above.

In the remaining part of this section, it will give some basic definitions and five lemmas so that the main conclusions can be expediently explained in the ensuing sections. 
Definition 1. [19] If there exists a compact set $\Omega \in \mathscr{R}^{n}$ such that $\forall s \in\left[t_{0}-h, t_{0}\right], \forall x(s) \in$ $\mathscr{R}^{n} \backslash \Omega, \varlimsup_{t \rightarrow+\infty} \rho(x(t), \Omega)=0$, then $\Omega$ is said to be a globally attractive set of (1), where $\mathscr{R}^{n} \backslash \Omega$ is the complement set of $\Omega$. A set $\Omega$ is called positive invariant set of (1), if $\forall s \in\left[t_{0}-h, t_{0}\right], \forall x(s) \in \mathscr{R}^{n} \backslash \Omega$ implies $x(t) \in \Omega$ for $t \geqslant t_{0}$.

Definition 2. [19] If there exists a radially unbounded and positive definite Lyapunov function $V(x(t))$, which satisfies $V(x(t)) \geqslant\|x\|^{\widetilde{\beta}}$, where $\widetilde{\beta}>$ 0 is a constant, and constants $\zeta>0, \beta>0$, such that for $V\left(x\left(t_{0}\right)\right)>\zeta, V(x(t))>\zeta, t \geqslant t_{0}$, the inequality $V(x(t))-\zeta \leqslant\left(\bar{V}\left(x\left(t_{0}\right)\right)-\zeta\right) \exp \left\{-\beta\left(t-t_{0}\right)\right\}$ always holds. Then, $\{x \mid V(x(t)) \leqslant \zeta\}$ is said to be a globally exponentially attractive set of (1), where $\bar{V}\left(x\left(t_{0}\right)\right) \geqslant V\left(x\left(t_{0}\right)\right)$ and $V\left(x\left(t_{0}\right)\right)$ is a constant.

Definition 3. CGNNs (1) with globally exponentially attractive set is said to be globally exponentially stable in Lagrange sense. CGNNs (1) with globally attractive set is said to be ultimately bounded.

Lemma 1. Let $a, b \in R^{n}, Y$ be a positive definite matrix, then the following inequality holds

$$
\pm 2 a^{T} b \leqslant a^{T} Y^{-1} a+b^{T} Y b .
$$

Proof. Since $Y>0$, it can obtain $a^{T} Y a \pm 2 a^{T} b+$ $b^{T} Y^{-1} b=\left(Y^{1 / 2} a \pm Y^{-1 / 2} b\right)^{T}\left(Y^{1 / 2} a \pm Y^{-1 / 2} b\right) \geqslant 0$. From this, it can easily obtain the inequality of Lemma 1.

Lemma 2. (Jensen's Inequality [28]) For any constant matrix $P \in R^{n \times n}, P^{T}=P>0, \quad \gamma>$ 0 , vector function $\omega:[0, \gamma] \rightarrow R^{n}$ such that the integrations concerned are well defined,then $\left(\int_{0}^{\gamma} \omega(s) \mathrm{d} s\right)^{T} P\left(\int_{0}^{\gamma} \omega(s) \mathrm{d} s\right) \leqslant \gamma \int_{0}^{\gamma} \omega^{T}(s) P \omega(s) \mathrm{d} s$.

Lemma 3. (Schur Complement [29]) Given constant matrices $P, Q$ and $R$, where $P^{T}=P, Q^{T}=Q$, then the following LMI:

$$
\left(\begin{array}{cc}
P & R \\
R^{T} & Q
\end{array}\right)<0
$$

is equivalent to the following conditions:
(1) $Q<0, P-R Q^{-1} R^{T}<0$;

(2) $P<0, Q-R^{T} P^{-1} R<0$.

Lemma 4. (Halanay Inequality [30]) Assume there exist $r_{1}>r_{2}>0$ and a nonnegative continuous quantity function $x(t)$, which satisfies $D^{+} x(t) \leqslant-r_{1} x(t)+r_{2} \bar{x}(t)$, for all $t \in\left[t_{0}-h, t_{0}\right]$, then $x(t) \leqslant \bar{x}\left(t_{0}\right) \exp \left(-\lambda\left(t-t_{0}\right)\right)$ holds for $\forall t \geqslant t_{0}$, where $\bar{x}(t)=\sup _{t-h \leqslant s \leqslant t} x(s), h \geqslant 0$, and $\lambda$ is the unique positive root of $\lambda=r_{1}-r_{2} e^{\lambda h}$.

The following lemma provides a key step in proving the main results of Lagrange stability for the CGNNs with mixed delays (1).

Lemma 5. Given constant matrices $A_{1}, A_{2}, A_{3}, B_{1}, B_{2}, B_{3} \in R^{n \times n}$, and appropriate reversible matrices $X, Y, Z$, let

$$
\begin{array}{r}
\Sigma_{1}=\left(\begin{array}{c}
A_{1} \\
B_{1}
\end{array}\right) X^{-1}\left(\begin{array}{l}
A_{1} \\
B_{1}
\end{array}\right)^{T}+\left(\begin{array}{l}
A_{2} \\
B_{2}
\end{array}\right) Y^{-1}\left(\begin{array}{l}
A_{2} \\
B_{2}
\end{array}\right)^{T} \\
+\left(\begin{array}{c}
A_{3} \\
B_{3}
\end{array}\right) Z^{-1}\left(\begin{array}{c}
A_{3} \\
B_{3}
\end{array}\right)^{T}, \\
\Sigma_{2}=\left(\begin{array}{lll}
A_{1} & A_{2} & A_{3} \\
B_{1} & B_{2} & B_{3}
\end{array}\right)\left(\begin{array}{lll}
X^{-1} & 0 & 0 \\
0 & Y^{-1} & 0 \\
0 & 0 & Z^{-1}
\end{array}\right)^{T} \\
\\
\left(\begin{array}{lll}
A_{1} & A_{2} & A_{3} \\
B_{1} & B_{2} & B_{3}
\end{array}\right)^{T},
\end{array}
$$

then $\Sigma_{1}=\Sigma_{2}$.

Proof. Firstly, we discuss $\Sigma_{1}$, and

$$
\begin{aligned}
& \Sigma_{1}=\left(\begin{array}{c}
A_{1} X^{-1} \\
B_{1} X^{-1}
\end{array}\right)\left(\begin{array}{c}
A_{1} \\
B_{1}
\end{array}\right)^{T}+\left(\begin{array}{c}
A_{2} Y^{-1} \\
B_{2} Y^{-1}
\end{array}\right)\left(\begin{array}{c}
A_{2} \\
B_{2}
\end{array}\right)^{T} \\
&+\left(\begin{array}{c}
A_{3} Z^{-1} \\
B_{3} Z^{-1}
\end{array}\right)\left(\begin{array}{c}
A_{3} \\
B_{3}
\end{array}\right)^{T} \\
&=\left(\begin{array}{cc}
A_{1} X^{-1} A_{1}^{T} & A_{1} X^{-1} B_{1}^{T} \\
B_{1} X^{-1} A_{1}^{T} & B_{1} X^{-1} B_{1}^{T}
\end{array}\right) \\
&+\left(\begin{array}{cc}
A_{2} Y^{-1} A_{2}^{T} & A_{2} Y^{-1} B_{2}^{T} \\
B_{2} Y^{-1} A_{2}^{T} & B_{2} Y^{-1} B_{2}^{T}
\end{array}\right) \\
&+\left(\begin{array}{ccc}
A_{3} Z^{-1} A_{3}^{T} & A_{3} Z^{-1} B_{3}^{T} \\
B_{3} Z^{-1} A_{3}^{T} & B_{3} Z^{-1} B_{3}^{T}
\end{array}\right)=\left(\begin{array}{ll}
\Delta_{1} & \Delta_{2} \\
\Delta_{2}^{T} & \Delta_{3}
\end{array}\right),
\end{aligned}
$$

where $\Delta_{1}=A_{1} X^{-1} A_{1}^{T}+A_{2} Y^{-1} A_{2}^{T}+A_{3} Z^{-1} A_{3}^{T}, \Delta_{2}=$ $A_{1} X^{-1} B_{1}^{T}+A_{2} Y^{-1} B_{2}^{T}+A_{3} Z^{-1} B_{3}^{T}, \Delta_{3}=B_{1} X^{-1} B_{1}^{T}+$ $B_{2} Y^{-1} B_{2}^{T}+B_{3} Z^{-1} B_{3}^{T}$. 
Calculating $\Sigma_{2}$, we obtain

$$
\begin{aligned}
& \Sigma_{2}=\left(\begin{array}{lll}
A_{1} & A_{2} & A_{3} \\
B_{1} & B_{2} & B_{3}
\end{array}\right)\left(\begin{array}{lll}
X^{-1} & 0 & 0 \\
0 & Y^{-1} & 0 \\
0 & 0 & Z^{-1}
\end{array}\right) \\
& \left(\begin{array}{ll}
A_{1}^{T} & B_{1}^{T} \\
A_{2}^{T} & B_{2}^{T} \\
A_{3}^{T} & B_{3}^{T}
\end{array}\right) \\
& =\left(\begin{array}{ccc}
A_{1} X^{-1} & A_{2} Y^{-1} & A_{3} Z^{-1} \\
B_{1} X^{-1} & B_{2} Y^{-1} & B_{3} Z^{-1}
\end{array}\right)\left(\begin{array}{cc}
A_{1}^{T} & B_{1}^{T} \\
A_{2}^{T} & B_{2}^{T} \\
A_{3}^{T} & B_{3}^{T}
\end{array}\right) \\
& =\left(\begin{array}{cc}
\Delta_{1} & \Delta_{2} \\
\Delta_{2}^{T} & \Delta_{3}
\end{array}\right) \text {. }
\end{aligned}
$$

Comparing the above equations, we can know $\Sigma_{1}=\Sigma_{2}$. The proof is finished.

\section{Main Results}

In this section, we will give some sufficient conditions for global exponential stability in Lagrange sense of CGNNs (1) in accordance with the lemmas in Section 2.

Theorem 6. Under assumption (A), the CGNNs system (1) is globally exponentially stable in Lagrange sense if there exist five positive diagonal matrices $P, Q, R, S, T$ and a positive definite matrix $H \in R^{n \times n}$ such that the following LMIs hold:

$$
\left(\begin{array}{ccccc}
\Theta_{11} & \Theta_{12} & \Theta_{13} & \Theta_{14} & P-L Q \\
* & \Theta_{22} & Q B & Q C & Q \\
* & * & -S & 0 & 0 \\
* & * & * & -T & 0 \\
* & * & * & * & -H
\end{array}\right)<0
$$

$$
W S W \leqslant P,
$$

where $\Theta_{11}=\alpha^{-1}(P+Q(F-L))-P D-D P+$ $2 L Q D+W\left(R+\sigma^{2} T\right) W, \quad \Theta_{12}=P A-L Q A-$ $D Q, \Theta_{13}=P B-L Q B, \Theta_{14}=P C-L Q C, \Theta_{22}=$ $Q A+A^{T} Q-R, \underline{\alpha}=\operatorname{diag}\left\{\alpha_{1}^{-}, \alpha_{2}^{-}, \ldots, \alpha_{\mathrm{n}}^{-}\right\}, \bar{\alpha}=$ $\operatorname{diag}\left\{\alpha_{1}^{+}, \alpha_{2}^{+}, \ldots, \alpha_{\mathrm{n}}^{+}\right\}, W=\operatorname{diag}\left\{\mathrm{w}_{1}, \mathrm{w}_{2}, \ldots, \mathrm{w}_{\mathrm{n}}\right\}$, $w_{i}=\max \left\{\left|L_{i}\right|,\left|F_{i}\right|\right\}, \forall i=1,2, \ldots, n$. Moreover, the set $\Omega=\left\{x \in R^{n} \mid x^{T}(t) \bar{\alpha}^{-1} P x(t) \leqslant \frac{U^{T} H U}{\varepsilon}\right\}$ is a globally exponentially attractive set and positive invariant set of system (1), where $0<\varepsilon \ll 1$.
Proof. We consider the following radially unbounded and positive definite Lyapunov functional with the given positive definite diagonal matrices $P=\operatorname{diag}\left\{\mathrm{p}_{1}, \ldots, \mathrm{p}_{\mathrm{n}}\right\}$ and $Q=\operatorname{diag}\left\{\mathrm{q}_{1}, \ldots, \mathrm{q}_{\mathrm{n}}\right\}$,

$$
\begin{aligned}
& V(x(t))=2 \sum_{i=1}^{n} p_{i} \int_{0}^{x_{i}(t)} \frac{s}{\alpha_{i}(s)} d s \\
& +2 \sum_{i=1}^{n} q_{i} \int_{0}^{x_{i}(t)} \frac{1}{\alpha_{i}(s)}\left(g_{i}(s)-L_{i} s\right) d s .
\end{aligned}
$$

Calculating the derivative of $V(x(t))$ along the positive semi-trajectory of (1), we can obtain

$$
\begin{aligned}
& \left.\frac{d V(x(t))}{d t}\right|_{(1)} \leqslant 2 x^{T}(t) P[-D x(t)+A g(x(t)) \\
& \left.+B g(x(t-\tau(t)))+C \int_{t-\sigma(t)}^{t} g(x(s)) d s+U\right] \\
& +2(g(x(t))-L x(t))^{T} Q[-D x(t)+A g(x(t)) \\
& \left.+B g(x(t-\tau(t)))+C \int_{t-\sigma(t)}^{t} g(x(s)) d s+U\right]= \\
& 2\left(x^{T}(t) P+g^{T}(x(t)) Q-x^{T}(t) L Q\right) \\
& (-D x(t)+A g(x(t))) \\
& +2\left(x^{T}(t) P B+g^{T}(x(t)) Q B-x^{T}(t) L Q B\right) \\
& g(x(t-\tau(t))) \\
& +2\left(x^{T}(t) P C+g^{T}(x(t)) Q C-x^{T}(t) L Q C\right) \\
& \int_{t-\sigma(t)}^{t} g(x(s)) d s \\
& +2\left(x^{T}(t) P+g^{T}(x(t)) Q-x^{T}(t) L Q\right) U .
\end{aligned}
$$

From assumption (A), for given positive diagonal matrix $R$ we derive

$$
\begin{aligned}
& 2\left(x^{T}(t) P+g^{T}(x(t)) Q-x^{T}(t) L Q\right) \\
& (-D x(t)+A g(x(t))) \\
& \leqslant 2 x^{T}(t)(-P D+L Q D) x(t) \\
& +2 g^{T}(x(t)) Q A g(x(t)) \\
& +2 x^{T}(t)(P A-L Q A-Q D) g(x(t)) \\
& +x^{T}(t) W R W x(t)-g^{T}(x(t)) \operatorname{Rg}(x(t))= \\
& \left(\begin{array}{c}
x(t) \\
g(x(t))
\end{array}\right)^{T}\left(\begin{array}{cc}
\Upsilon & \Theta_{12} \\
* & \Theta_{22}
\end{array}\right)\left(\begin{array}{c}
x(t) \\
g(x(t))
\end{array}\right)
\end{aligned}
$$

where $\Upsilon=-P D-D P+2 L Q D+W R W$.

By using assumption (A), Lemma 1 and Lemma 2, we know that there exist two positive diagonal 
matrices $S, T$ and a positive definite matrix $H$ such that the following inequalities hold

$$
\begin{aligned}
& 2\left(x^{T}(t) P B+g^{T}(x(t)) Q B-x^{T}(t) L Q B\right) \\
& g(x(t-\tau(t))) \leqslant \\
& \left(x^{T}(t) P B+g^{T}(x(t)) Q B-x^{T}(t) L Q B\right) S^{-1} \\
& \left(x^{T}(t) P B+g^{T}(x(t)) Q B-x^{T}(t) L Q B\right)^{T} \\
& +g^{T}(x(t-\tau(t))) S g(x(t-\tau(t))) \\
& \leqslant\left(\begin{array}{c}
x(t) \\
g(x(t))
\end{array}\right)^{T}\left(\begin{array}{c}
(P-L Q) B \\
Q B
\end{array}\right) S^{-1} \\
& \left(\begin{array}{c}
(P-L Q) B \\
Q B
\end{array}\right)^{T}\left(\begin{array}{c}
x(t) \\
g(x(t))
\end{array}\right) \\
& +x^{T}(t-\tau(t)) W S W x(t-\tau(t)), \\
& 2\left(x^{T}(t) P C+g^{T}(x(t)) Q C-x^{T}(t) L Q C\right) \\
& \int_{t-\sigma(t)}^{t} g(x(s)) d s \leqslant \\
& \left(x^{T}(t) P C+g^{T}(x(t)) Q C-x^{T}(t) L Q C\right) T^{-1} \\
& \left(x^{T}(t) P C+g^{T}(x(t)) Q C-x^{T}(t) L Q C\right)^{T} \\
& +\left(\int_{t-\sigma(t)}^{t} g(x(s)) d s\right)^{T} T\left(\int_{t-\sigma(t)}^{t} g(x(s)) d s\right) \\
& \leqslant\left(\begin{array}{c}
x(t) \\
g(x(t))
\end{array}\right)^{T}\left(\begin{array}{c}
(P-L Q) C \\
Q C
\end{array}\right) T^{-1} \\
& \left(\begin{array}{c}
(P-L Q) C \\
Q C
\end{array}\right)^{T}\left(\begin{array}{c}
x(t) \\
g(x(t))
\end{array}\right) \\
& +\sigma^{2} x^{T}(t) W T W x(t), \\
& 2\left(x^{T}(t) P+g^{T}(x(t)) Q-x^{T}(t) L Q\right) U \\
& \leqslant\left(x^{T}(t) P+g^{T}(x(t)) Q-x^{T}(t) L Q\right) H^{-1} \\
& \left(x^{T}(t) P+g^{T}(x(t)) Q-x^{T}(t) L Q\right)^{T}+U^{T} H U \\
& =\left(\begin{array}{c}
x(t) \\
g(x(t))
\end{array}\right)^{T}\left(\begin{array}{c}
P-L Q \\
Q
\end{array}\right) H^{-1} \\
& \left(\begin{array}{c}
P-L Q \\
Q
\end{array}\right)^{T}\left(\begin{array}{c}
x(t) \\
g(x(t))
\end{array}\right)+U^{T} H U \text {. }
\end{aligned}
$$

Based on Lemma 5 and (5)-(9), we have

$$
\begin{aligned}
& \left.\frac{d V(x(t))}{d t}\right|_{(1)} \leqslant\left(\begin{array}{c}
x(t) \\
g(x(t))
\end{array}\right)^{T} \Pi\left(\begin{array}{c}
x(t) \\
g(x(t))
\end{array}\right) \\
& +\left(\begin{array}{c}
x(t) \\
g(x(t))
\end{array}\right)^{T} \Lambda\left(\begin{array}{c}
x(t) \\
g(x(t))
\end{array}\right) \\
& +x^{T}(t-\tau(t)) W S W x(t-\tau(t))+U^{T} H U,
\end{aligned}
$$

where

$$
\begin{aligned}
& \Pi=\left(\begin{array}{cc}
\Upsilon+\sigma^{2} W T W & \Theta_{12} \\
* & \Theta_{22}
\end{array}\right), \\
& \Lambda=\left(\begin{array}{ccc}
(P-L Q) B & (P-L Q) C & P-L Q \\
Q B & Q C & Q
\end{array}\right) \\
& \left(\begin{array}{ccc}
S^{-1} & 0 & 0 \\
0 & T^{-1} & 0 \\
0 & 0 & H^{-1}
\end{array}\right)\left(\begin{array}{cc}
B^{T}(P-L Q) & B^{T} Q \\
C^{T}(P-L Q) & C^{T} Q \\
P-L Q & Q
\end{array}\right) .
\end{aligned}
$$

Following from (2), there exists $0<\varepsilon \ll 1$ such that

$$
\left(\begin{array}{ccccc}
\widetilde{\Theta} & \Theta_{12} & \Theta_{13} & \Theta_{14} & P-L Q \\
* & \Theta_{22} & Q B & Q C & Q \\
* & * & -S & 0 & 0 \\
* & * & * & -T & 0 \\
* & * & * & * & -H
\end{array}\right)<0
$$

where $\widetilde{\Theta}=(1+\varepsilon) \underline{\alpha}^{-1}(P+Q(F-L))-P D-D P+$ $2 L Q D+W\left(R+\sigma^{2} T\right) W$. In the light of Lemma 3, one gets

$$
\left(\begin{array}{cc}
\widetilde{\Theta} & \Theta_{12} \\
* & \Theta_{22}
\end{array}\right)+\Lambda<0
$$

meanwhile, it is noticed that

$$
\left(\begin{array}{cc}
\widetilde{\Theta} & \Theta_{12} \\
* & \Theta_{22}
\end{array}\right)=\Pi+\left(\begin{array}{cc}
M & 0 \\
0 & 0
\end{array}\right) .
$$

where $M=(1+\varepsilon) \underline{\alpha}^{-1}(P+Q(F-L))$. Therefore, it can be deduced that

$$
\Pi+\Lambda<\left(\begin{array}{cc}
-(1+\varepsilon) \underline{\alpha}^{-1}(P+Q(F-L)) & 0 \\
0 & 0
\end{array}\right) .
$$

Combining (3), (10) and (11), we can derive

$$
\begin{aligned}
& \left.\frac{d V(x(t))}{d t}\right|_{(1)} \leqslant \\
& -(1+\varepsilon) x^{T}(t) \underline{\alpha}^{-1}(P+Q(F-L)) x(t) \\
& +x^{T}(t-\tau(t)) P x(t-\tau(t))+U^{T} H U, t \geqslant t(12)
\end{aligned}
$$

From assumption (A) and the formula (4), one has

$V(x(t)) \leqslant x^{T}(t) \underline{\alpha}^{-1}(P+Q(F-L)) x(t), t \geqslant t_{0}$. 
According to (12) and (13), we obtain

$$
\begin{aligned}
& \left.\frac{d V(x(t))}{d t}\right|_{(1)} \leqslant-(1+\varepsilon) V(x(t))+\bar{V}(x(t)) \\
& +U^{T} H U, \quad t \geqslant t_{0},
\end{aligned}
$$

where $\bar{V}(x(t))=\sup _{t-h \leqslant s \leqslant t} V(s)$.

On the basis of (14), when $V(x(t))>$ $\eta, \bar{V}(x(t))>\eta$, one gets

$$
\begin{aligned}
& \left.\frac{d(V(x(t))-\eta)}{d t}\right|_{(1)} \leqslant-(1+\varepsilon)(V(x(t))-\eta) \\
& +(\bar{V}(x(t))-\eta), \quad t \geqslant t_{0},
\end{aligned}
$$

where $\eta=\frac{U^{T} H U}{\varepsilon}$. According to Lemma 4, we are able to derive $(V(x(t))-\eta) \leqslant \bar{V}((x(t))-$ $\eta) \exp (-\lambda t)$, where $\lambda$ is the unique positive root of $\lambda=(1+\varepsilon)-e^{\lambda h}$. Simultaneously, judging by [31], it is easy to prove that there exists a constant $\widetilde{\beta}$ such that $V(x(t)) \geqslant\|x\|^{\widetilde{\beta}}$. In terms of Definition 1, Definition 2 and noticing $V(x(t)) \geqslant x^{T}(t) \bar{\alpha}^{-1} P x(t)$, it is said that $\Omega=\left\{x \in R^{n} \mid x^{T}(t) \bar{\alpha}^{-1} P x(t) \leqslant \frac{U^{T} H U}{\varepsilon}\right\}$ is a globally exponentially attractive set and positive invariant set of system (1). Hence, the CGNNs system (1) is globally exponentially stable in Lagrange sense via the Definition 3. So, the proof of Theorem 6 is completed.

Remark 2. Obviously, in light of Definition 3, it is known that if the network (1) have a global attractive set, it is ultimately bounded; and if the network (1) has a globally exponentially attractive set, it is globally exponentially stable in Lagrange sense. Furthermore, the latter include the former. Hence, the study of Lagrange stability depends on the existence of global exponentially attractive set, not considering uniformly bounded of the system. However, in the reference[18, 21-25], the Lagrange stability was determined by both uniformly bounded and globally exponentially attractive set.

Remark 3. We denote $\Sigma=\{\psi \in C(R, R) \mid s \psi(s)>$ $0, s \neq 0$, and $\left.D^{+} \psi(s) \geqslant 0, s \in R\right\}$. Four different types of activation functions are listed as follows:

(H1) $g(\cdot) \in \Gamma_{1}, \quad$ where $\Gamma_{1}=\left\{g(\cdot) \mid g_{i} \in\right.$ $C(R, R), \quad \exists k_{i}>0, \quad\left|g_{i}\left(x_{i}\right)\right| \leqslant k_{i}, \quad \forall x_{i} \in R, \quad i=$
$1,2, \cdots, n\}$, and $g(\cdot)$ is the bounded continuous function.

(H2) $g(\cdot) \in \Gamma_{2}$, where $\Gamma_{2}=\left\{g(\cdot) \mid g_{i} \in \Sigma, \quad \exists k_{i}>\right.$ $\left.0, \quad x_{i} g_{i}\left(x_{i}\right) \leqslant k_{i} x_{i}^{2}, \quad \forall x_{i} \in R, \quad i=1,2, \cdots, n\right\}$, and $g(\cdot)$ is the Lurie-type function.

(H3) $g(\cdot) \in \Sigma$, where $g(\cdot)$ is the continuous nondecreasing function.

(H4) $g(\cdot) \in \Gamma_{3}$, where $\Gamma_{3}=\left\{g(\cdot) \mid g_{i} \in \Sigma, \quad \exists k_{i}>\right.$ $0, \quad\left|g_{i}(x)-g_{i}(y)\right| \leqslant k_{i}|x-y|, \quad \forall x_{i} \in R, \quad i=$ $1,2, \cdots, n\}$, and $g(\cdot)$ is the Lipschitz-type function.

In $[18,19,21,22,24,25]$, the scholars studied the global stability in Lagrange sense of neural networks with both time-varying delays and three different types of activation functions which include (H1)-(H3). Owing to the constants $L_{i}, F_{i}$ in assumption (A) being arbitrary, the condition about activation function of this paper is weaker than $(\mathrm{H} 1),(\mathrm{H} 2)$ and (H4). It is also observed that assumption (A) and (H3) don't embody each other.

Corollary 7. Under assumptions (A) and let $\underline{\alpha}=$ $\operatorname{diag}\left\{\alpha_{1}^{-}, \ldots, \alpha_{\mathrm{n}}^{-}\right\}, \bar{\alpha}=\operatorname{diag}\left\{\alpha_{1}^{+}, \ldots, \alpha_{\mathrm{n}}^{+}\right\}, \sigma(\overline{\mathrm{t}})=$ 0 . The CGNNs system (1) is globally exponentially stable in Lagrange sense if there exist four positive diagonal matrices $P, Q, R, S$ and a positive definite matrix $H \in R^{n \times n}$ such that the following LMIs hold:

$$
\left.\begin{array}{cccc}
\Xi & \Theta_{12} & \Theta_{13} & P-L Q \\
* & \Theta_{22} & Q B & Q \\
* & * & -S & 0 \\
* & * & * & -H
\end{array}\right)<0,
$$

where $\Xi=\alpha^{-1}(P+Q(F-L))-P D-D P+$ $2 L Q D+W R W, \quad W=\operatorname{diag}\left\{\mathrm{w}_{1}, \ldots, \mathrm{w}_{\mathrm{n}}\right\}, \quad w_{i}=$ $\max \left\{\left|L_{i}\right|,\left|F_{i}\right|\right\}, \forall i=1,2, \ldots, n$. Moreover, the set $\Omega=\left\{x \in R^{n} \mid x^{T}(t) \bar{\alpha}^{-1} P x(t) \leqslant \frac{U^{T} H U}{\varepsilon}\right\}$ is a globally exponentially attractive set and positive invariant set of system (1), where $0<\varepsilon \ll 1$.

Proof. The course of proof is almost parallel to that of Theorem 6, except for the inequality (8) in the theorem 6 . So the proof is omitted in here.

In the CGNNs system (1), if let $\alpha(t) \equiv 1$, the system (1) will degenerate into the following mixed delayed recurrent neural network which is described 
by the matrix form:

$$
\begin{aligned}
& \dot{x}(t)=-D x(t)+A g(x(t))+B g(x(t-\tau(t))) \\
& +C \int_{t-\sigma(t)}^{t} g(x(s)) d s+U .
\end{aligned}
$$

Similarly, there also exist the following results.

Theorem 8. Under assumption (A), the system (18) is globally exponentially stable in Lagrange sense if there exist two positive definite matrices $P, H \in R^{n \times n}$ and four positive diagonal matrices $Q, R, S, T$ such that the following LMIs hold:

$$
\left(\begin{array}{ccccc}
\Xi_{1} & \Theta_{12} & \Theta_{13} & \Theta_{14} & P-L Q \\
* & \Theta_{22} & Q B & Q C & Q \\
* & * & -S & 0 & 0 \\
* & * & * & -T & 0 \\
* & * & * & * & -H
\end{array}\right)<0,
$$

where $\Xi_{1}=P+Q(F-L)-P D-D P+2 L Q D+$ $W\left(R+\sigma^{2} T\right) W, W=\operatorname{diag}\left\{\mathrm{w}_{1}, \mathrm{w}_{2}, \ldots, \mathrm{w}_{\mathrm{n}}\right\}, w_{i}=$ $\max \left\{\left|L_{i}\right|,\left|F_{i}\right|\right\}, \forall i=1,2, \ldots, n$. Moreover, the set $\Omega_{1}=\left\{x \in R^{n} \mid x^{T}(t) P x(t) \leqslant \frac{U^{T} H U}{\varepsilon}\right\}$ is a globally exponentially attractive set and positive invariant set of system (18), where $0<\varepsilon \ll 1$.

Proof. Chosen the Lyapunov functional $V_{1}(x(t))=$ $x^{T}(t) P x(t)+2 \sum_{i=1}^{n} q_{i} \int_{0}^{x_{i}(t)}\left(g_{i}(s)-L_{i} s\right) d s$, transformed LMIs (2) and (3) into LMIs (19) and (20), and used a similar technique to compute the derivative of $V_{1}(x(t))$ along the positive half trajectory of (18), the next course of proof is analogous to that of Theorem 8 , so it is omitted in here.

Remark 4. When assuming $\sigma(t)=0$ in the theorem 8 , it could be found that the conclusion of theorem 8 in this paper will turn to the main result of Theorem 1 in [23] right now.

In particular, when $H$ is elected as a positive diagonal matrix in Theorem 8 and let $\sigma(t)=0$. Based on (19) and by virtue of Lemma 3, one gets

$$
\begin{aligned}
& \left(\begin{array}{ccc}
\Xi_{2} & P A-L Q A-D Q & P B-L Q B \\
* & Q A+A^{T} Q-R & Q B \\
* & * & -S
\end{array}\right) \\
& +\left(\begin{array}{c}
P-L Q \\
Q \\
0
\end{array}\right) H^{-1}\left(\begin{array}{c}
P-L Q \\
Q \\
0
\end{array}\right)^{T}<Q 21
\end{aligned}
$$

where $\Xi_{2}=P+Q(F-L)-P D-D P+2 L Q D+$ $W R W$. Owing to $H$ is a positive diagonal matrix, so $H^{-1}$ is a positive diagonal matrix too. Therefore, from (21) and on the basis of S-produce method [29], one gains

$$
\left(\begin{array}{ccc}
\Xi_{2} & P A-L Q A-D Q & P B-L Q B \\
* & Q A+A^{T} Q-R & Q B \\
* & * & -S
\end{array}\right)<0 .
$$

Then it is known that the network (18) has a unique equilibrium point (see [27]). As a result, the following corollary is obtained.

Corollary 9. Under assumption (A) and if there exist four positive diagonal matrices $Q, R, S, H$ and $a$ positive definite matrix $P \in R^{n \times n}$ such that the following LMIs hold:

$$
\left(\begin{array}{cccc}
\Xi_{2} & \Theta_{12} & \Theta_{13} & P-L Q \\
* & \Theta_{22} & Q B & Q \\
* & * & -S & 0 \\
* & * & * & -H
\end{array}\right)<0,
$$

where $W=\operatorname{diag}\left\{\mathrm{w}_{1}, \mathrm{w}_{2}, \ldots, \mathrm{w}_{\mathrm{n}}\right\}, \quad$ and $w_{i}=$ $\max \left\{\left|L_{i}\right|,\left|F_{i}\right|\right\}, \forall i=1,2, \ldots, n$. Then the network (18) is globally exponentially stable in Lyapunov sense.

Remark 5. Corollary 9 in this paper is the main result of Theorem 1 in [27].

\section{Qualitative study on the trajectory of CGNNs (1) in the complementary set of the globally exponential attracting set $\Phi$}

In this section, we only present a theorem to illustrate the application of the globally exponentially attractive set and positive invariant set. Because the equilibrium points, periodic solution, chaotic attractor and almost periodic solution of Eq.(1) or Eq.(24) are all positive invariant sets. So, as a direct application of the results obtained in the previous, we prove for general case of positive invariant set, more details see the following theorem. 
Theorem 10. Let $\Phi$ is a globally exponentially attractive set and positive invariant set of CGNNs (1), then in the complementary set $R^{3} / \Phi$, (i.e., outside of the globally exponentially attractive set $\Phi)$, there are no bounded positive invariant sets that do not intersect $\Phi$.

Proof. Suppose this is not true. Without loss of generality, assume that $\Psi$ is a positive invariant set of $R^{3} / \Phi$. Then $\Psi \cup \Phi=\phi$, where $\phi$ denotes the empty set. This implies that $\Phi$ and $\Psi$ do not intersect. So we have $\inf _{y \in \Phi, x \in \Psi}\|x-y\|>0$. From the definition of positive invariant set, we know that $x\left(t, t_{0}, x_{0}\right) \in \Psi$, for $x_{0} \in \Psi$ and $t \geqslant t_{0}$. Hence,

$$
\inf _{y \in \Phi, x\left(t, t_{0}, x_{0}\right) \in \Psi, t>t_{0}}\left\|y-x\left(t, t_{0}, x_{0}\right)\right\|>0 .
$$

On the other hand, since $\Phi$ is a globally exponentially attractive set, we have that $x\left(t, t_{0}, x_{0}\right) \rightarrow \Phi$ for any $x_{0} \in R^{3}$ as $t \rightarrow+\infty$, which implies that

$$
\inf _{y \in \Phi, x\left(t, t_{0}, x_{0}\right) \in \Psi, t>t_{0}}\left\|y-x\left(t, t_{0}, x_{0}\right)\right\|=0,
$$

leads to a contradiction to the above inequality. This shows that the conclusion of Theorem 10 is true.

\section{Illustrative Examples}

In this section, we will give two numerical examples to verify our theoretical results with different activation functions.

Example 1. Consider a three-neuron CGNNs system (1) with the following parameters:

$\alpha(x(t))=2+0.5 \cos (x(t)), \tau(t)=0.8|\sin (t)|, \delta(t)=$ $2|\cos (t)|, U=(0.8862,0.6455,0.9312)^{T}$,

$D=\left(\begin{array}{ccc}3.5 & 0 & 0 \\ 0 & 6.2 & 0 \\ 0 & 0 & 4.3\end{array}\right)$,

$A=\left(\begin{array}{ccc}8 & -2 & 5 \\ -8 & 6 & 9 \\ 4 & 2 & -5\end{array}\right), B=\left(\begin{array}{ccc}2 & -5 & 1 \\ 4 & 8 & 5 \\ 7 & -6 & -7\end{array}\right)$,

$C=\left(\begin{array}{ccc}-1.32 & 0.34 & -0.55 \\ -0.10 & 0.13 & 0.28 \\ 0.02 & 0.36 & 0.75\end{array}\right)$.

Case 1: When the activation function is selected as $g(x(t))=\frac{1}{16}(x(t)+\tanh (x(t)))$, it is obvious that the activation function $g(\cdot)$ satisfies assumption (A) with $L=0$,

$F=W=\left(\begin{array}{ccc}0.125 & 0 & 0 \\ 0 & 0.125 & 0 \\ 0 & 0 & 0.125\end{array}\right)$.

Then we calculate the parameters $\alpha_{1}^{+}=\alpha_{2}^{+}=\alpha_{3}^{+}=$ $2.5, \alpha_{1}^{-}=\alpha_{2}^{-}=\alpha_{3}^{-}=1.5$.

So, $\bar{\alpha}^{-1}=\left(\begin{array}{ccc}0.4 & 0 & 0 \\ 0 & 0.4 & 0 \\ 0 & 0 & 0.4\end{array}\right)$,

$\underline{\alpha}^{-1}=\left(\begin{array}{ccc}0.667 & 0 & 0 \\ 0 & 0.667 & 0 \\ 0 & 0 & 0.667\end{array}\right)$.

By using the Matlab LMI Control Toolbox, the solutions to the LMIs in (2) and (3) are derived as follows:

$$
\begin{aligned}
P & =\left(\begin{array}{ccc}
3.7705 & 0 & 0 \\
0 & 4.3831 & 0 \\
0 & 0 & 3.6559
\end{array}\right), \\
Q & =\left(\begin{array}{ccc}
0.7541 & 0 & 0 \\
0 & 0.7091 & 0 \\
0 & 0 & 2.7052
\end{array}\right), \\
R & =\left(\begin{array}{ccc}
275.0812 & 0 & 0 \\
0 & 144.3950 & 0 \\
0 & 0 & 113.1999
\end{array}\right), \\
S & =\left(\begin{array}{ccc}
122.8730 & 0 & 0 \\
0 & 216.0459 & 0 \\
0 & 0 & 134.5125
\end{array}\right), \\
T & =\left(\begin{array}{ccc}
43.7955 & 0 & 0 \\
0 & 60.0178 & 0 \\
0 & 0 & 50.1016
\end{array}\right), \\
H & =\left(\begin{array}{ccc}
87.4051 & -1.1089 & 1.7740 \\
-1.1089 & 86.2600 & -0.8219 \\
1.7740 & -0.8219 & 86.5896
\end{array}\right) .
\end{aligned}
$$

Calculating the eigenvalues of $H$, we get the eigenvalues are 85.1731, 85.6617, 89.4199. Therefore, following from Theorem 6, we gain that the network (1) is globally exponentially stable in Lagrange sense, and the set $\Omega=\left\{x \in R^{n} \mid 1.5082 x_{1}^{2}+\right.$ $\left.1.7532 x_{2}^{2}+1.4624 x_{3}^{2} \leqslant \frac{180.1224}{\varepsilon}\right\}$ is a globally exponentially attractive set and positive invariant set of (1), where $0<\varepsilon \ll 1$. However, according to the theorem 3.2 of the reference [25], we gain 


$$
\begin{aligned}
& \Sigma_{1}=\left(\begin{array}{ccccc}
-15 & -2 & 5 & 1 & -2.5 \\
8 & -38.6 & 9 & 2 & 4 \\
4 & 2 & -34.4 & 3.5 & -3 \\
1 & -2.5 & 0.5 & -0.2 & 0 \\
2 & 4 & 2.5 & 0 & -0.2 \\
3.5 & -3 & -3.5 & 0 & 0 \\
-0.66 & 0.17 & -0.275 & 0 & 0 \\
-0.05 & -0.065 & 0.14 & 0 & 0 \\
0.01 & 0.18 & 0.375 & 0 & 0
\end{array}\right. \\
& \begin{array}{llll}
0.5 & -0.66 & 0.17 & -0.275
\end{array} \\
& \begin{array}{llll}
2.5 & -0.05 & -0.065 & 0.14
\end{array} \\
& \begin{array}{llll}
-3.5 & 0.01 & 0.18 & 0.375
\end{array} \\
& \left.\begin{array}{cccc}
0 & 0 & 0 & 0 \\
0 & 0 & 0 & 0 \\
-0.2 & 0 & 0 & 0 \\
0 & 3 & 0 & 0 \\
0 & 0 & 3 & 0 \\
0 & 0 & 0 & 3
\end{array}\right) .
\end{aligned}
$$

By calculating we get the six eigenvalues of $\Sigma_{1}$ are $-41.3715,-32.5883,-13.6091,-0.6148+0.5381 \mathrm{i}$, $-0.6148-0.5381 \mathrm{i}, 0.1755,3.0172,3.0059,3.0000$, from which we are able to know that is not negative definite. So the conclusion in [25] can't be applied to ensure the global exponential stability in Lagrange sense of (1). Hence, for this case, our results in this paper are less conservative than those in [25].

Case 2: On the other hand, let $\alpha(t) \equiv 1$ and chose $g(x(t))=\frac{1}{16}(|x+1|-|x-1|)$, the activation function $g(\cdot)$ satisfies assumption (A) with

$$
\begin{aligned}
& L=\left(\begin{array}{ccc}
-0.125 & 0 & 0 \\
0 & -0.125 & 0 \\
0 & 0 & -0.125
\end{array}\right), \\
& F=W=\left(\begin{array}{ccc}
0.125 & 0 & 0 \\
0 & 0.125 & 0 \\
0 & 0 & 0.125
\end{array}\right) .
\end{aligned}
$$

Analogously, by using the Matlab LMI Control Toolbox, the solutions to the LMIs in (19) and (20) are derived as follows:

$$
\begin{aligned}
& P=\left(\begin{array}{ccc}
5.2500 & 0.9791 & -2.1937 \\
0.9791 & 5.8322 & 1.1810 \\
-2.1937 & 1.1810 & 7.4673
\end{array}\right), \\
& Q=\left(\begin{array}{ccc}
1.9109 & 0 & 0 \\
0 & 1.6459 & 0 \\
0 & 0 & 4.1828
\end{array}\right),
\end{aligned}
$$

$$
\begin{aligned}
R & =\left(\begin{array}{ccc}
260.7605 & 0 & 0 \\
0 & 190.7273 & 0 \\
0 & 0 & 194.4384
\end{array}\right), \\
S & =\left(\begin{array}{ccc}
153.7660 & 0 & 0 \\
0 & 183.9017 & 0 \\
0 & 0 & 138.0816
\end{array}\right), \\
T & =\left(\begin{array}{ccc}
51.0309 & 0 & 0 \\
0 & 77.4338 & 0 \\
0 & 0 & 75.3789
\end{array}\right), \\
H & =\left(\begin{array}{ccc}
104.2528 & -0.1554 & 0.2436 \\
-0.1554 & 103.6230 & 0.5247 \\
0.2436 & 0.5247 & 104.1278
\end{array}\right) .
\end{aligned}
$$

Calculating the eigenvalues of $P$ and $H$, we get the eigenvalues of $P$ are $3.1123,6.5290,8.9081$ and that of $H$ are 103.2528, 104.4315, 105.0220. Therefore, following from Theorem 8, we gain that the network (18) is globally exponentially stable in Lagrange sense, and the set $\Omega=\left\{x \in R^{n} \mid\right.$ $\left.3.1123 x_{1}^{2}+6.5290 x_{2}^{2}+8.9081 x_{3}^{2} \leqslant \frac{215.6712}{\varepsilon}\right\}$ is a globally exponentially attractive set and positive invariant set of (18), where $0<\varepsilon \ll 1$.

Remark 6. The activation functions in Case 1 of this example don't meet with $\left(H_{1}\right),\left(H_{2}\right)$ or $\left(H_{3}\right)$, which means that the conclusion in $[18,19,21,22$, $24,25]$ can't be applied to ensure the global exponential stability in Lagrange sense of (1). Hence, our results in this paper are less conservative than those in $[18,19,21,22,24,25]$.

Example 2. Considering the following two-neuron recurrent neural networks with time-varying delay:

$$
\dot{x}(t)=-D x(t)+A g(x(t))+B g(x(t-\tau(t)))+U,
$$

where $\tau(t)=0.8|\sin (t)|, U=(1,1.5)^{T}$,

$D=\left(\begin{array}{cc}3.5 & 0 \\ 0 & 6.2\end{array}\right), A=\left(\begin{array}{cc}8 & -2 \\ 4 & 2\end{array}\right)$,

$B=\left(\begin{array}{cc}2 & -5 \\ 4 & 8\end{array}\right)$.

Case 1: We consider the activation function $g(x(t))=\frac{1}{8}(|x+1|-|x-1|)$. In this case, the activation function $g(\cdot)$ satisfies assumption (A) with $L=\left(\begin{array}{cc}-0.25 & 0 \\ 0 & -0.25\end{array}\right), F=W=\left(\begin{array}{cc}0.25 & 0 \\ 0 & 0.25\end{array}\right)$. 
Analogously, the solutions are derived as follows:

$P=\left(\begin{array}{ll}3.2329 & 0.2169 \\ 0.2169 & 6.4594\end{array}\right), Q=\left(\begin{array}{cc}1.7007 & 0 \\ 0 & 1.7889\end{array}\right)$,

$R=\left(\begin{array}{cc}134.3960 & 0 \\ 0 & 89.2271\end{array}\right)$,

$S=\left(\begin{array}{cc}41.9425 & 0 \\ 0 & 86.8791\end{array}\right)$,

$H=\left(\begin{array}{cc}63.4544 & 0.0122 \\ 0.0122 & 62.7065\end{array}\right)$.

Calculating the eigenvalues of $P$ and $H$, we get the eigenvalues of $P$ are 3.2184, 6.4739 and that of $H$ are 63.4546, 62.7063. Therefore, following from Theorem 8, we gain that the network (18) is globally exponentially stable in Lagrange sense, and the set $\Omega=\left\{x \in R^{n} \mid 3.2184 x_{1}^{2}+6.4739 x_{2}^{2} \leqslant \frac{204.5438}{\varepsilon}\right\}$ is a globally exponentially attractive set and positive invariant set of (24), where $0<\varepsilon \ll 1$.

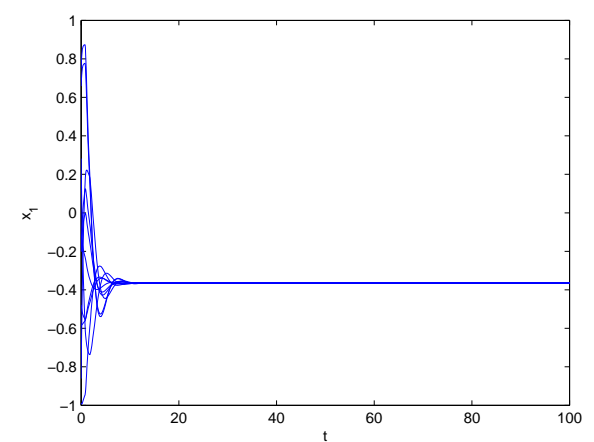

Fig.1. State curves $x_{1}(t)$ of system (24) with activation function $g(x(t))=\frac{1}{8}(|x+1|-|x-1|)$.

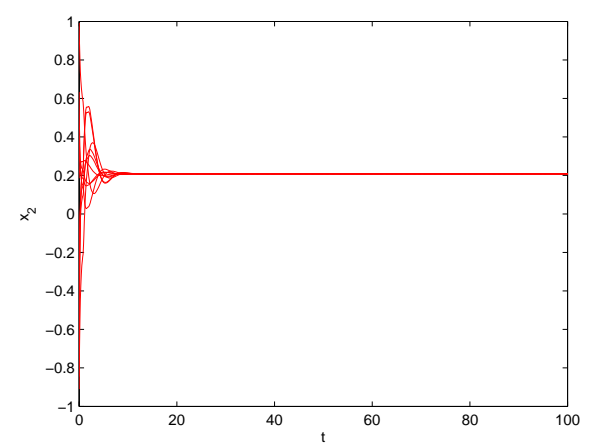

Fig.2. State curves $x_{2}(t)$ of system (24) with activation function

$$
g(x(t))=\frac{1}{8}(|x+1|-|x-1|) .
$$

Remark 7. From Corollary 9, we can know that the network (24) has a unique equilibrium point which is globally exponentially stable. In fact, the global exponential stability in Lyapunov sense of the unique equilibrium point is demonstrated in Fig.1 and Fig. 2 which are ten trajectories of state $x_{1}(t)$ and $x_{2}(t)$ of the system (24) with random initial conditions.

Case 2: If we choose the activation functions as $g(x(t))=\frac{1}{5}(x(t)+\tanh (x(t)))$, it is obviously that the activation function $g(\cdot)$ satisfies assumption (A) with $L=0, F=W=\left(\begin{array}{cc}0.4 & 0 \\ 0 & 0.4\end{array}\right)$.

Then by using the Matlab LMI Control Toolbox, the solutions are derived as follows:

$P=\left(\begin{array}{cc}5.2632 & 0.2579 \\ 0.2579 & 9.0456\end{array}\right), Q=\left(\begin{array}{cc}1.0160 & 0 \\ 0 & 1.7402\end{array}\right)$,
$R=\left(\begin{array}{cc}170.0008 & 0 \\ 0 & 115.4947\end{array}\right)$,
$S=\left(\begin{array}{cc}73.5609 & 0 \\ 0 & 143.4947\end{array}\right)$,
$H=\left(\begin{array}{cc}79.0877 & -0.3404 \\ -0.3404 & 78.1530\end{array}\right)$.

Calculating the eigenvalues of $P$ and $H$, we get the eigenvalues of $P$ are 5.2457, 9.0631 and that of $H$ are $79.1985,78.0421$. Therefore, following from Theorem 8, we gain that the network (24) is globally exponentially stable in the Lagrange sense, and the set $\Omega=\left\{x \in R^{n} \mid 5.2457 x_{1}^{2}+9.0631 x_{2}^{2} \leqslant \frac{254.7932}{\varepsilon}\right\}$ is a globally exponentially attractive set and positive invariant set of (24), where $0<\varepsilon \ll 1$. However, according to the theorem 4.2 of the reference [18], we gain

$Q^{(1)}=\left(\begin{array}{cccc}9 & -2 & 1 & -2.5 \\ 4 & 3 & 2 & 4 \\ 1 & -2.5 & -1 & 0 \\ 2 & 4 & 0 & -1\end{array}\right)$.

By calculating we get the four eigenvalues of $Q^{(1)}$ are 7.1618+3.3577i, 7.1618-3.3577i, -1.6052, 2.7183 , from which we are able to know that $Q^{(1)}$ is not negative definite. So the conclusion in [18] can't be applied to ensure the global exponential stability in Lagrange sense of (24). Hence, for this example, our results in this paper are less conservative than those in [18].

The following figures are given to testify the validity of the results. Fig.3 shows the state trajectories of system (24) with $g(x(t))=\frac{1}{5}(x+\tanh (x))$ and Fig.4 shows the behavior of system (24) with 
ten state trajectories of random initials.

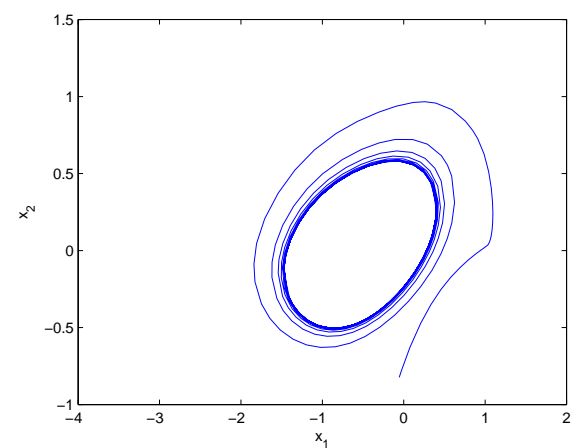

Fig.3. The trajectories of state of system (24) with activation function $g(x(t))=\frac{1}{5}(x+\tanh (x))$ and arbitrary initial condition.

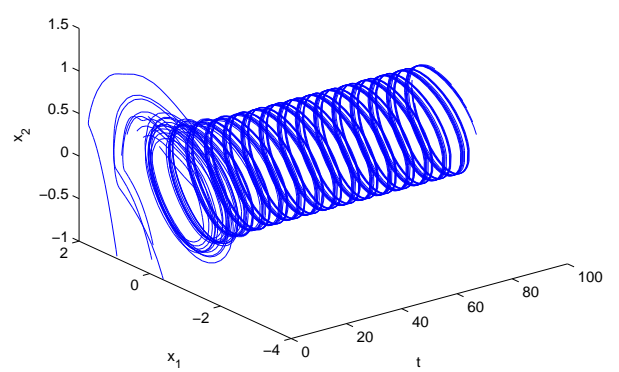

Fig.4. The behavior of system (24) with ten state trajectories of random initials.

\section{Conclusions}

In this paper, we have investigated the Lagrange stability problem for a class of Cohen-Grossberg neural networks with general activation functions and mixed time delays. The mixed time delays considered here are time-varying delays and finite distributed delays. Based on assuming that the activation functions are neither bounded nor monotonous or differentiable, a set of novel algebra criteria for global exponential stability in Lagrange sense of CGNNs are obtained by virtue of Lyapunov functional, Halanay delay differential inequality and linear matrix inequality. Obviously, our approaches are different from those of the pre-existing, and more importantly, the obtain results extend and generalize that of the works [18, 23, 25, 27]. Meanwhile, our results also show that the globally exponentially attractive set does contribute to the Lagrange stability of the considered system. Yet again, it is verified that outside the global exponential attractive set, there is no periodic state, almost periodic state or chaos attractor. These Lagrange stability analysis can narrow the search domains of optimization and associative memories, and provide theoretical guidelines for applications. Finally, we give two examples to show the effectiveness of our theoretical results.

\section{Acknowledgments}

The authors would like to thank the anonymous reviewers for their helpful comments and suggestions. This work is supported by National Natural Science Foundation of China under Grant No.60774036, Natural Science Foundation of Hubei Province of China (2008CDA063) and Research Fund for Central Colleges (C2009Z025Y).

\section{References}

1. M.A. Cohen and S. Grossberg, "Absolute stability of global pattern formation and parallel memory storage by competitive neural networks," IEEE Trans. Syst. Man Cybern., 13, 815-826 (1983).

2. Z.S. Wang and H.G. Zhang, "Global asymptotic stability of reaction-diffusion Cohen-Grossberg neural networks with continuously distributed delays," IEEE trans. neural netw., 21 (1), 39-49 (2010).

3. Z.S. Wang, H.G. Zhang and P. Li, "An LMI approach to stability analysis of reaction-diffusion Cohen-Grossberg neural networks concerning dirichlet boundary conditions and distributed delays," IEEE trans. Syst. Man Cyber., 40 (6), 1596-1606 (2010).

4. P. Balasubramaniam and M. Syed Ali, "Robust exponential stability of uncertain fuzzy Cohen-Grossberg neural networks with time-varying delays," Fuzzy Sets Syst., 161, 608-618 (2010).

5. Q.X. Zhu and J.D. Cao, "Robust exponential stablility of Markovian Jump impulsive stochastic CohenGrossberg neural networks with mixed time delays," IEEE Trans. Neural Netw., 21 (8), 1314-1325 (2010).

6. C.X. Huang and J.D. Cao, "Covergence dynamics of stochastic Cohen-Grossberg neural networks with unbounded distributed delays," IEEE trans. neural netw. 22 (4), 561-572 (2011).

7. T.W. Huang, "Robust stability of delayed fuzzy Cohen-Grossberg neural networks," Comput. Math. Appl., 61 (8), 2247-2250 (2011).

8. J.L. Wang, H.N. Wu and L. Guo, "Stability analysis of reaction-diffusion Cohen-Grossberg neural 
networks under impulsive control," Neurocomputing, http://dx.doi.org/10.1016/j.neucom.2012.11.006.

9. C. Zhou, H. Zhang, H. Zhang and C. Dang, "Global exponential stability of impulsive fuzzy CohenGrossberg neural networks with mixed delays and reaction-diffusion terms," Neurocomputing, 91, 67-76 (2012).

10. D. Li, D. He and D.Y. Xu, "Mean square exponential stability of impulsive stochastic reaction-diffusion Cohen-Grossberg neural networks with delays," Math. Comput. Simulation, 82, 1531-1543 (2012).

11. Y.Zhang and Q.Luo, "Novel stability criteria for impulsive delayed reaction-diffusion Cohen-Grossberg neural networks via Hardy-Poincarè inequality," Chaos Solitons Fractals, 45, 1033-1040 (2012).

12. D.H. He and X.H. Wang, "Attracting and invariant sets of impulsive delay Cohen-Grossberg neural networks," Nonlinear Anal. HS, 6, 705-711 (2012).

13. Z.V. Rekasius, "Lagrange stability of nonlinear feedback systems," IEEE Trans. Automat. Control., 8 (2), 160-163 (1963).

14. K.W. Thornton and R.J. Mulholland, "Lagrange stability and ecological systems," J. Theor. Biol., 45, 473-485 (1974).

15. K.M. Passino and K.L. Burgess, "Lagrange stability and boundedness of discrete event systems," Discrete Event Dyn. Syst., 5, 383-403 (1995).

16. J.Z. Wang, Z.S. Duan and L. Huang, "Control of a class of pendulum-like systems with Lagrange stability," Automatica, 42, 145-150 (2006).

17. Y. Yang and L. Huang, "Lagrange stability of a class of nonlinear discreted time system," in: First IEEE Conference on Industrial Electronics and Applications, 1-6 (May 2006).

18. X.X. Liao, Q. Luo, Z.G Zeng and Y.X. Guo, "Global exponential stability in Lagrange sense for recurrent neural networks with time delays," Nonlinear Anal. RWA, 9, 1535-1557 (2008).

19. X.X. Liao, Q. Luo and Z.G. Zeng, "Positive invariant and global exponential attractive sets of neural networks with time-varying delays," Neurocomputing, 71, 513-518 (2008).

20. Y.Q. Yang and J.D. Cao, "A feedback neural network for solving convex constraint optimization problems,"
Appl. Math. Comput., 201, 340-350 (2008).

21. Q. Luo, Z.G. Zeng and X.X. Liao, "Global exponential stability in Lagrange sense for neutral type recurrent neural networks," Neurocomputing, 74, 638-645 (2011).

22. A.L. Wu, Z.G. Zeng, C.J. Fu and W.W. Shen, "Global exponential stability in Lagrange sense for periodic neural networks with various activation functions," Neurocomputing, 74, 831-837 (2011).

23. Z.W. Tu, J.G. Jian and K. Wang, "Global exponential stability in Lagrange sense for recurrent neural networks with both time-varying delays and general activation functions via LMI approach," Nonlinear Anal. RWA, 12 (4), 2174-2182 (2011).

24. X.H. Wang, M.H. Jiang and S.L. Fang, "Stability analysis in Lagrange sense for a non-autonomous CohenGrossberg neural network with mixed delays," Nonlinear Anal. TMA, 70, 4294-4306 (2009).

25. B.X. Wang, J.G. Jian and M.H. Jiang, "Stability in Lagrange sense for Cohen-Grossberg neural networks with time-varying delays and finite distributed delays," Nonlinear Anal. Hybrid Syst., 4, 65-78 (2010).

26. P. Gahinet, A. Nemirovski, M. Laub and Chilali, "LMI control toolbox user's guide", The Mathworks, Massachusetts, 1995.

27. Q.K. Song, "Exponential stability of recurrent neural networks with both time-varying delays and general activation functions via LMI approach," Neurocomputing, 71, 2823-2830 (2008).

28. K. Gu, V. Kharitonov and J. Chen, "Stability of timedelay systems," Birkhauser, Boston, MA, 2003.

29. B. Boyd, L.E. Ghoui, E. Feron and V. Balakrishnan, "Linear Matrix Inequalities in System and Control Theory," SIAM, Philadelphia, PA, 1994.

30. A. Halanay, "Differential Equations: Stability, Oscillations, Time Lags, Academic Press," New York, 1966.

31. X.X. Liao, Y. Fu and S. Xie, "Globally exponential stability of Hopfied networks," Adv. Syst. Sci. Appl., 5, 533-545 (2005).

32. G.J. Wang, J.D. Cao and L. Wan, "Global dissipativity of stochastic neural networks with time delay," $J$. Franklin Inst., 346, 794-807 (2009). 